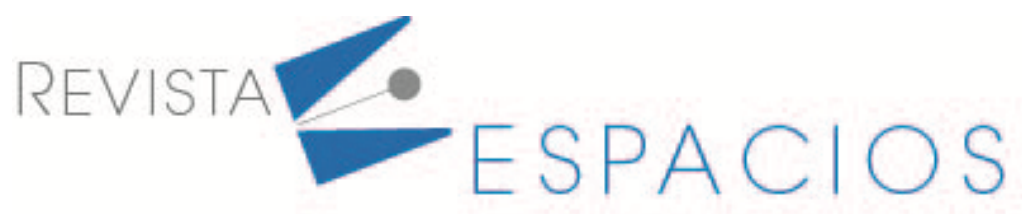

\title{
Calidad de la educación, a propósito de la voz de los maestros con respecto a la relación entre la escuela, el contexto y el currículo
}

\section{Quality of education, ownership of teachers' voices regarding the relationship between school, context and curriculum}

PINO MONTOYA, José Wilmar ${ }^{1}$

GALLEGO HENAO, Adriana María ${ }^{2}$

PELAEZ HENAO, Oscar Alberto ${ }^{3}$

ARROYAVE TABORDA, Leisy Magdalli ${ }^{4}$

CASTAÑO GÓMEZ, Melina ${ }^{5}$

\begin{abstract}
Resumen: Este artículo es resultado de la investigación: "La gestión académica como estrategia para la construcción de calidad educativa: una mirada narrativa desde los maestros". Objetivo comprender la gestión académico pedagógica de los docentes y su relación con la calidad educativa. La metodología fue cualitativa y la estrategia el estudio de caso. Se encontró que la calidad educativa depende de los actores, contextos y procesos institucionales. Se llega a la conclusión que los procesos de calidad están permeados por condiciones particulares de la escuela.

Palabras claves: calidad de la educación, currículo, escuela rural, personal docente, admiración educativa.
\end{abstract}

\begin{abstract}
This article is the result of the research: "Academic management as a strategy for the construction of educational quality: a narrative view from teachers". Objective is to understand the academic pedagogical management of teachers and its relationship with educational quality. The methodology was qualitative and the strategy the case study. It was found that educational quality depends on the actors, contexts and institutional processes. It is concluded that the quality processes are permeated by particular conditions of the school.

Keywords: quality of education, curriculum, rural school, teaching staff, educational admiration.
\end{abstract}

\section{Introducción}

Entender la calidad como un proceso dinámico y frente al cual cada uno de los personajes de la realidad escolar tiene algo que decir, es un asunto difícil por cuanto el concepto de calidad se soporta en conceptualizaciones

\footnotetext{
${ }^{1}$ Estudiante de Posdoctorado en Bioética; Doctor en Filosofía, Magister en Educación y Desarrollo Humano. Docente investigador, Universidad Católica Luis Amigó.Jose.pinomo@amigo.edu.co.

${ }^{2}$ Magíster en Educación y Desarrollo Humano, Especialista en Docencia investigativa Universitaria, Licenciada en Educación Preescolar. Docente investigadora y coordinadora Maestría en Educación, Universidad Católica Luis Amigó. Adriana.gallegohe@amigo.edu.co

${ }^{3}$ Magíster en Educación, Especialista en la enseñanza Lenguas Extrajeras, Licenciado en Educación religiosa. Docente investigador y coordinador del campo investigativo Licenciatura en Inglés, Universidad Católica Luis Amigó. Oscar.pelaezhe@amigo.edu.co

${ }^{4}$ Magíster en Adicciones, Licenciada en Educación Básica. Docente investigadora y coordinadora de las Especializaciones en Gestión Educativa y Docencia Universitaria. leisy.arroyaveta@amigo.edu.co

${ }^{5}$ Politóloga. Auxiliar de investigación. Melicas17@gmail.com
} 
generales, surgidos desde la perspectiva empresarial de producto final y de eficiencia (Aguerrondo, 1993), que luego, según Gálvez, (2005) fue trasladada a campos sociales, como la educación, el mismo que "se ha visto reflejado en las diferentes políticas educativas regionales, nacionales e internacionales con las cuales se busca orientar los procesos educativos de niños, niñas y adolescentes". (Gallego, Peláez, Pino, Gonzales y Arroyave, 2020 , p. 1). Lo que ha propuesto un tipo de calidad fundamentado en el logro de resultados de los alumnos que se evidencia en las diferentes pruebas objetivas de corte nacional e internacional como las Pisa y las pruebas Saber Pro y las Icfes.

No obstante, la pregunta por la calidad educativa poco o nada incluye o implica la construcción desde el diálogo de saberes, la suma de las discusiones y las posiciones de todos; y desde allí construir de manera colectiva las líneas de referencia asociadas al concepto de calidad, los aspectos a considerar y los cuestionamientos que soportan la reflexión de los docentes al respecto.

En esta misma línea, el concepto tradicional de calidad educativa no tiene sus principios en el acercamiento a la escuela, a sus realidades y a las personas que conviven en su interior; ni mucho menos convoca la pregunta por las relaciones culturales, por los espacios y rutinas que allí se comparten, pero más que nada por el sentido que imprimen los individuos a sus discursos y prácticas. Es aquí, en donde pueden rastrearse los saberes que respaldan sus acciones y las comprensiones desde las cuales es posible seguir la construcción del concepto de calidad desde el contexto, escuela y el currículo

Con este interés se propone y en alianza entre la Universidad Católica Luis Amigó y el municipio de Santa Rosa de Osos el estudio: "La gestión académica como estrategia para la construcción de calidad educativa: una mirada narrativa desde los maestros", cuyas preguntas de investigación fueron: ¿Cómo práctican los docentes la gestión académica y cómo estas le aportan a la calidad educativa? ¿Qué concepto tienen los maestros de la calidad educativa? ¿Desde la escuela y su contexto cómo se le aporta a la calidad de la educación? De allí que el objetivo general es comprender la gestión académico pedagógica de los docentes y su relación con la calidad educativa de la Institución Educativa San Isidro del Municipio de Santa Rosa de Osos del departamento de Antioquia.

Esta investigación, reconoce en los diferentes participantes actores fundamentales para entender lo que sucede alrededor de la calidad educativa en el aula. Se trata entonces, de hacer una construcción colectiva en la que no existen barreras conceptuales entre investigadores e investigados; toda vez que se parte del principio de la corresponsabilidad conceptual en la que todos los participantes aportan en la consolidación de los hallazgos y sus interpretaciones, teniendo como horizonte un proceso de investigación plural y transversal. Por ello, construcciones como el currículo, la figura del docente y el lugar del contexto en el proceso escolar son tratados en el marco del concepto de calidad y de las prácticas que dan cuenta de este, ello además de lograr articular el trabajo desde lo metodológico permite exponer la realidad de los participantes desde sus testimonios, sus tensiones alrededor del proceso de enseñanza aprendizaje y los desafíos que asumen en clave de nombrar lo que hacen y aventurarse en la interpretación de sus acciones.

Finalmente, el texto comparte los resultados generales de la investigación, señalando cuestionamientos frente al concepto de calidad educativa en cabeza del Estado, la sociedad y los propios docentes; buscando con ello mostrar que la categoría de calidad en la educación es el lugar común de la escuela, del quehacer del profesor relacionado con el contexto; pero que por la incapacidad de pormenorizar sus distintos componentes y asumir responsabilidades de fondo, termina siendo tratada desde la generalización, el discurso político y las tecnologías; o peor aún, desde la utopía desdibujada de las clases menos favorecidas y en este caso desde el contexto y la situación de la población rural. 


\section{Metodología}

La investigación se inscribe en el paradigma cualitativo en el cual "El diseño es abierto tanto a lo que concierne a la selección de participantes actuantes, como en lo que hace referencia a la interpretación y análisis. El investigador como sujeto de investigación integra los contextos situacional y convencional (...)". (Galeano, 2004, p. 28) y tiene como objeto acercarse a las comprensiones que tienen los sujetos en torno a las acciones que realizan desde los procesos educativos que acompañan.

Resulta clave para el cumplimiento de los propósitos de la investigación señalar la práctica pedagógica como epicentro de la labor docente, entender sus dinámicas, los saberes que imbrica, son algunas de las razones que validan la decisión de optar por el paradigma cualitativo. En este sentido Canales (2006) establece que:

(...) el investigador cualitativo se mueve en el orden de los significados y sus reglas de significación: los códigos y los documentos, o significaciones. Metodológicamente el punto es cómo posibilitar una reproducción de la comunidad o colectivo de hablantes de una lengua común para su análisis y comprensión. (p. 19)

En este estudio fue vital la comprensión de la práctica y la reconstrucción del sentido que los sujetos dan a esta en un contexto en particular. Llevarlo a cabo, implica el acercamiento minucioso, casi artesanal, a la cotidianidad en la que discurre el objeto de estudio y los sujetos que lo hacen posible. De allí que se optó por el estudio de caso, el cual pretende y, en palabras de Króll (2004) “(...) mantener la unidad del todo, [es] el esfuerzo por no perder el carácter unitario de la entidad que está siendo estudiada (un individuo, una organización, una cultura, etcétera)". (p. 256). Por otra parte, en esta investigación fueron claves los elementos que permean la cotidianidad de la escuela rural y para su entendimiento se apeló a las voces de los participantes, para desde allí construir los datos y sus análisis, lo que implicó el uso de técnicas que permitan la disertación, el encuentro en la palabra y la construcción colectiva y se eligió como técnica de recolección de información la entrevista estructurada. "La entrevista estructurada se diferencia de la no estructurada en que ésta es preparada con anterioridad por el investigador, con la intención de llevar a cabo un orden determinado durante el encuentro con su(s) informante(s)". (López, 2002, p. 227-228)

La población con la que se desarrolló la investigación está compuesta por 9 docentes de la institución Educativa San Isidro de la zona Rural del municipio de Santa Rosa de Osos (Antioquia). El análisis de los datos se hizo en tres etapas, la primera la codificación de los testimonios con el fin de repetir el anonimato del entrevistado. Ver tabla 1.

Tabla 1

Codificación de las entrevistas

\begin{tabular}{|c|c|c|}
\hline Docente & Instrumento & Código \\
\hline 1 & Entrevista 1 & D1 \\
\hline 2 & Entrevista 2 & D2 \\
\hline 3 & Entrevista 3 & D3 \\
\hline 4 & Entrevista 4 & D4 \\
\hline 5 & Entrevista 5 & D5 \\
\hline 6 & Entrevista 6 & D6 \\
\hline 7 & Entrevista 7 & D7 \\
\hline 8 & Entrevista 8 & D8 \\
\hline 9 & Entrevista 9 & D9 \\
\hline
\end{tabular}

Fuente: Elaboración propia 
La segunda etapa fue la clasificación por categorías de análisis; y la tercera la contrastación de los testimonios con los referentes teóricos, para lo cual se utilizó una matriz de análisis categorial; posterior se construyó el informe final del que se obtuvo como resultado el artículo propuesto.

En coherencia con el paradigma cualitativo, la investigación hace devoluciones a los participantes a través de dos productos; el primero la divulgación de este artículo científico de la investigación; y el segundo, un evento académico de divulgación en el marco del foro educativo de la municipalidad 2019 en la que se socializó a los docentes los resultados parciales de esta investigación.

\section{Resultados}

Acercarse a la escuela, a sus dinámicas, a las personas que la habitan, los espacios que ocupa y su historia implica volver a los principios, lineamientos, propósitos y tareas que la sociedad históricamente ha legado a esta institución. Es regresar a los cimientos que soportan el ejercicio didáctico y pedagógico de los docentes, es retornar las motivaciones de los estudiantes $y$, por supuesto, las condiciones necesarias para lograr las metas altruistas y, en ocasiones, hasta utópicas que se asocian a la acción escolar. En este orden de ideas, el concepto de calidad educativa es un término difícil de concretar ya que es "casi intangible, variable, dinámico, que cambia con el tiempo y con las personas que lo definen (...); ciertamente no es fácil llegar a un consenso sobre lo que supone la calidad en la educación" (Casanova, 2012, p. 8), es entonces un concepto polisémico que, a pesar de su inexactitud, ha cobrado gran importancia en los discursos educativos en las últimas décadas.

Partiendo del argumento anterior, se precisaron los hallazgos y se brindará la discusión con relación a lo que se encontró de la calidad educativa en la institución San Isidro.

\subsection{Calidad educativa y su relación con el contexto}

El sociólogo francés Pierre Bourdieu (1997) acuñó durante mediados del siglo XX el concepto de Capital Cultural, el cual hoy puede entenderse como el conjunto de adelantos, costumbres, posiciones éticas y saberes que la sociedad resguarda como parte del patrimonio que como especie ha escogido preservar y compartir; tarea que, en buena parte, ha sido dejada a la escuela y que la escuela como institución ampliamente ha reglado, parametrizado y amoldado a niveles, grados, espacios y títulos. Un concepto de calidad está relacionado con el rescate del capital cultural, enmarcado en la aplicabilidad de los enseñado en el contexto en el que se desenvuelve el estudiante.

(...) ese concepto de calidad, es no solo que el muchacho sepa, que uno le dé las herramientas, que sepa, (además) qué necesita el muchacho, (enseñarle) qué hacer con lo que sabe, qué aplicabilidad tiene eso que aprendió en su vida, en su contexto, en su cotidianidad, en su práctica social, si tiene un lenguaje adecuado, un léxico adecuado para dialogar para socializarse, para interactuar con el otro. (D3)

En este escenario, resulta fundamental entender cuáles son los dispositivos que la escuela ha escogido para cumplir la tarea de formar en el capital cultural de un grupo social. Uno de ellos y como se deja entrever en el siguiente testimonio, es adecuar los contenidos al contexto.

Para eso hay que tener dos cosas en cuenta: por un lado, el tema o la temática que se va dar; y por otro lado, hay que tener en cuenta el contexto porque por ejemplo acá estamos en una zona rural entonces hay que tener en cuenta de dónde vienen los niños, cuando hablamos de contexto hay que tener en cuenta que en las familias no hay un nivel académico muy alto entonces no se puede poner mucha investigación 
porque tampoco hay muchos medios de comunicación, no tienen acceso a internet, entonces se debe pensar qué ponemos como tarea para la casa y desde el colegio qué se puede hacer cuando estamos preparando la clase. (D8)

Como se observa, adecuar el contenido al contexto implica la reflexión sobre tres aspectos fundamentalmente: el profesor, entendido como el artesano; el contexto, asumido como el espacio de interacción, y el currículo como la manifestación concreta del capital cultural. En este sentido calidad educativa implica leer el contexto para adecuar el currículo a su necesidad. Así lo demuestra la Docente 9.

Pero siento que a veces he chocado con esos lineamientos nacionales, porque me estoy limitando más al contexto, a las necesidades de los chicos, entonces me centro más como en el contexto, tengo en cuenta el estándar, lo que dice, su profundidad, y de pronto cómo trabajar, pero... pero a veces importa más es como el aula misma, lo que uno se encuentra en el aula, qué es lo verdadero que está ahí, en el aula y este contexto que nos rodea (D9)

En este orden de ideas, el elemento que convoca la reflexión sobre la calidad en la escuela, como espacio de interacción y formación, es el contexto. El contexto históricamente ha estado ligado al espacio físico, en otras palabras, a la infraestructura escolar, sus formas y adecuaciones. Esta acepción del concepto convoca una dificultad que niega la incidencia de factores sociales, culturales, históricos y las representaciones como condicionantes determinantes en las maneras como se identifica y se piensa un espacio.

Se trata entonces, de hacer evidente que los entornos escolares están habitados por personas y son las interacciones, los discursos, las prácticas y los modos de vida de esas personas las que configuran un territorio escolar. Por tanto, hablar de entorno o contexto escolar convoca la polifonía que convive con la escuela, que la nutren y la hacen posible. A propósito de esto Restrepo (2017) establece:

El territorio no es simplemente lo que vemos; mucho más que montañas, ríos, valles, asentamientos humanos, puentes, caminos, cultivos, paisajes, es el espacio habitado por la memoria y la experiencia de los pueblos. Por eso aprender a leerlo y descifrarlo puede enseñar mucho sobre cómo resolver los problemas y los conflictos, las dudas y las incertidumbres que enfrentamos en el presente. (p.26)

Es de la interacción de estos elementos que emana la calidad escolar y es con estos elementos que la triada compuesta por docente-contexto-currículo se relaciona y lo complementa. En este caso se diría como argumenta Cano (1998) que "(...) entendemos calidad como el proceso consensuado (por todos los miembros implicados) de construcción de objetivos para cada contexto y momento y como la trayectoria o tendencia que realizamos para conseguirlos. (106).

La tarea escolar de asumir el contexto como un elemento esencial para lograr la calidad, convoca la pregunta por las personas que lo habitan y lo hacen posible. Bajo la premisa de que el contexto surge de la interacción, privilegiamos los usos y costumbres sobre los lugares y su destinación funcional; esto permite entender porqué un escenario deportivo puede ser un teatro, una pista de baile o un improvisado mercado para aprender las operaciones básicas.

Esta idea del entorno, como una construcción dinámica y permeada por las costumbres y modos particulares de las personas del territorio, permite a la escuela pensarse en clave de lo local, en favor de salvaguardar los saberes que allí son importantes y de formar en aquellos que se hacen necesarios. Es una apuesta por una formación en términos de pertinencia, derivada de una lectura minuciosa que hace la comunidad educativa y en la que, a manera de engranaje, se acoplan las distintas piezas que permiten el proceso escolar. 
Hemos señalado antes que la idea de contexto y las interacciones de los individuos que lo habitan convocan a la calidad educativa, pero también hay que tener presente los espacios ocupados por estos. Es precisamente este segundo elemento el espacio, uno de los que genera mayores contratiempos a la hora de valorar el cumplimiento de las metas que la escuela ha trazado, representando en sí mismo uno de los mayores obstáculos a enfrentar.

Esta afirmación es compartida por los diversos actores que participan de la escena educativa, un ejemplo de ello es la UNESCO (2016) quienes fruto del análisis de las pruebas TERCE ${ }^{6}$ en cuyo informe final incluyen el siguiente apartado:

En relación a la infraestructura, existe un amplio consenso que indica que las características de los espacios físicos de las escuelas son una condición necesaria para generar ambientes propicios para el aprendizaje y el desarrollo de habilidades, aunque por sí solas no generan mejor calidad, y deben estar acompañadas con políticas educativas que potencien el alcance de las inversiones en infraestructura escolar. (p.34)

La afirmación del Banco Interamericano de Desarrollo (BID) es clave para entender la relevancia de las políticas públicas como agentes fundamentales dentro del esquema educativo, lo encontrado en el TERCE muestra que la inversión en infraestructura educativa, va de la mano de políticas públicas que forman en su uso, que las proyectan a todos los actores comunitarios y que principalmente velan por su articulación al inventario existente, una especie de apropiación que permite la inserción del nuevo recurso dentro de las dinámicas de la comunidad escolar, en este línea uno de los participantes argumenta:

La calidad educativa pasa por la dotación, la infraestructura y el aprovechamiento. Es importante que las inversiones en educación posibiliten condiciones óptimas para el proceso de enseñanza con espacios pertinentes, docentes en permanente formación y con políticas públicas que incentiven la cualificación y estimulen el mejoramiento. (D5)

Lo anterior, expone no solo la necesidad de nuevos espacios para el aprendizaje o la adecuación de los ya existentes. Pone sobre la mesa, la necesidad de acompañar con formación, adecuación y padrinazgos a los establecimientos educativos para el uso de espacios, materiales y equipos que poco transitan por la realidad escolar, muchos de los cuales podrían impactar en gran medida los aprendizajes de los estudiantes y el desarrollo de las competencias.

\subsection{Escuela, docente y calidad educativa}

El proceso de formación está ligado a la figura de la persona que encara los procesos in-situ, es decir por el rostro de quienes llevan a cabo el proceso educativo y de aquellos que se convierten en el enlace de los otros dos componentes (contexto-currículo). El docente, maestro o profesor, agencia los pormenores de la tarea escolar y tal como lo establece la UNESCO (2014) en el documento sobre los objetivos para el desarrollo sostenible, constituye un actor determinante en el desarrollo de una sociedad, a propósito de lo anterior subraya:

Un sistema educativo es apenas tan bueno como sus docentes. Liberar su potencial es esencial para mejorar la calidad del aprendizaje. Todo indica que la calidad de la educación mejora cuando se apoya a los docentes y se deteriora en caso contrario, lo que contribuye a los alarmantes niveles de analfabetismo entre los jóvenes (p.37)

${ }^{6}$ Tercer Estudio Regional Comparativo y Explicado - TERCE 
De lo anterior resalta como la figura del profesor está asociada a la calidad educativa, a los logros del proceso de formación y al acompañamiento frente a vulnerabilidades tales como el analfabetismo, los grupos armados, la pobreza extrema y la explotación, por solo citar las más relevantes.

Esta postura está relacionada con las tareas que los docentes asumen dentro del proceso de construcción de los aprendizajes. Tareas en las que la planeación, la lectura de contexto e incluso el compromiso con los territorios hacen parte de la visión sobre el quehacer en el aula. A propósito de lo anterior uno de los participantes expone:

La tarea del docente desborda lo que pasa durante los minutos que dura la sesión de encuentro con los estudiantes. Es una acción constante que promueve la reflexión, el encuentro con la incertidumbre y con la cultura. De ahí que sea permanente la tarea del docente de aprender, reflexionar e investigar. (D4)

Múltiples investigaciones en Latinoamérica han evidenciado que existe una estrecha relación entre las prácticas pedagógicas y el cumplimiento de los propósitos asociados a un nivel educativo, aspecto que hace más importante la reflexión sobre el lugar del profesor, toda vez que es quien materializa la práctica pedagógica en el aula de clase y quien puede favorecer los aspectos que la constituyen reflexionado su propio que hacer. Como lo dice un docente:

Cada vez que salgo de una clase salgo triste o contenta ¿porque? Porque me reflexionó, porque yo al acabar la clase digo dios mío lo que hice todas las actividades que traje, igual bien y me preguntan lo mismo y hemos hecho este juego y hemos hecho esta actividad he hecho una evaluación y siguen tantos vacíos, qué es lo que me pasa, será que no estoy llevando la clase como debe de ser, porque de verdad me analizó demasiado y digo entonces que tengo que hacer de verdad para poder llegarle a estos estudiantes. (D3)

Como se evidencia, es el docente el que tiene incidencia directa en la práctica pedagógica y esa incidencia está permeada por su reflexión, experiencia y la lectura del territorio. De este modo, la reflexión esta mediada por las sensaciones que quedan en el profesor fruto del encuentro con el aula de clase y con sus estudiantes, es una especie de balance en el que es posible determinar las posibilidades, las limitantes y los escenarios de cualificación del proceso de enseñanza - aprendizaje. Pero adicionalmente, se constituye en la primera condición para la concreción del saber pedagógico, el cual, para Zambrano (2005), constituye el referente para explicar lo que pasa en el aula de clase, comprenderlo y resignificarlo. Es precisamente la reflexión, un elemento que es valorado por los docentes como un punto crucial en la comprensión del acto educativo y que adicionalmente ligan a los procesos de calidad.

Otro elemento relevante en la relación entre maestro y calidad educativa, es el que alude a su saber disciplinar, los planteamientos didácticos y los procesos de formación. Obsérvese lo dicho al respecto.

Sí, para mí, mi mejor escuela ha sido haber estudiado en la normal, o sea ser normalista, o sea un docente se hace allá, se hace un verdadero maestro, porque a usted allá le da las bases, de verdad yo digo, una persona que pasa por la universidad para mí qué yo lo viví en la Católica del Norte y eso que lo hice virtual, sino que tuve la oportunidad de que fuera de allá, se vuelve más mecánico en cambio para mí un docente el mejor estudio es la normal, yo allá hice mis prácticas, y desde allá. De verdad la formación que allá es exigente. (D3)

Empecemos por decir que el saber disciplinar es el eje de la labor del profesor, este se materializa en las decisiones sobre el que enseñar y en las competencias que asocia a esos ejes conceptuales; pero tal como lo señala Zambrano (2005) va más allá, en tanto involucra elementos de la vida del sujeto como su propia experiencia de formación y su lectura de la realidad cultural: 
En el saber disciplinar del profesor encontramos la práctica del gesto, el indicio y la competencia. El gesto, la forma como el profesor se dispone en actos de comprensión de lo que conoce, los indicios que lo llevan a dudar o reafirmar lo conocido y las competencias que surgen en los discursos de su saber marcan la distancia. Lo que caracteriza el saber disciplinar es la forma como el profesor es capaz de volver sobre lo que conoce, lo que domina del conocimiento que produce la disciplina donde ha sido formado. El saber disciplinar, además, es un tipo de saber cuyo concepto fundamental es la distancia y la reflexión. (p.55)

Y aquí es importante volver a otro de los elementos propuestos por Zambrano, la Distancia, que puede ser entendida como el proceso que vive el profesor entre el momento que adquiere un saber y el momento que lo agencia en el aula de clase, tiempo en el que ese saber sufre variaciones fruto de las experiencias, el reconocimiento de sus estudiantes y el encuentro con la realidad, y en el que el profesor determina los alcances de aquello que sabe y los aspectos en los que debe seguir avanzando. Esto puede verse reflejado en el siguiente testimonio:

El mejoramiento de lo que se hace en el aula de clase, pasa por dos condiciones: La primera la actualización permanente del docente y la segunda es su capacidad de aprender de lo hecho, es decir de su experiencia. De la unión de esas dos condiciones se fortalece su pedagogía y su didáctica. (D5)

Este docente asocia la calidad en los procesos educativos a la consolidación de un saber disciplinar y adicionalmente a la posibilidad de volver sobre la práctica con el ánimo de aprender de ella. Una suerte de ciclo investigativo, en donde el objeto de estudio es la práctica del docente y en el que la comprensión de lo que allí sucede, construye condiciones para la cualificación de la misma en términos pedagógicos y didácticos. En este momento es importante señalar, que no existe una dicotomía entre el saber disciplinar y el saber pedagógico; al contrario, son saberes complementarios que transitan de manera paralela el aula de clase: la acción del profesor y por supuesto su práctica. Conviene imaginar una especie de campo disciplinar, en el que convergen distintos elementos frente al propósito de la formación, el proceso enseñanza aprendizaje y la cultural escolar.

En este sentido es que la tarea de la escuela en la búsqueda de lograr la calidad educativa de resulta compleja, toda vez que implica trasgredir las barreras de lo físico, lo tangible y lo técnico para rastrear en los actores escolares las prácticas, los imaginarios y las posiciones que yacen en la cotidianidad de los sujetos, en sus conversaciones y actitudes habituales; esto puede verse con mayor claridad en el siguiente testimonio:

La clase va más allá de las paredes del aula, es necesario conocer los espacios del establecimiento educativo y las dinámicas de las personas que lo componen. Es importante saber de qué hablan las personas, las necesidades que tienen, saber hacia dónde quieren ir como comunidad. Es desde ese conocimiento que el docente parte para construir el proceso de enseñanza. (D1)

No basta entonces con conocer el lugar y las características geográficas del espacio, esta es tan solo la primera estación. El recorrido que hace la escuela para configurar la ruta enseñanza - aprendizaje, pasa por involucrar otras reflexiones sobre el acto de aprender; por ejemplo, los posicionamientos éticos y políticos ligados a la construcción de nuevos saberes, las formas tradicionales y ancestrales del saber para esa comunidad, por solo mencionar algunos.

\subsection{El currículo como manifestación del capital cultural}

Entre las tareas a la que se enfrenta la escuela en el marco de la gestión académica, están aquellas relacionadas con los procesos de planeación, desarrollo y cualificación de las prácticas escolares, este tipo de acciones están relacionadas con los saberes que transitan el espacio escolar, las relaciones de los individuos al interior del 
mismo, los conocimientos que se han privilegiado para la enseñanza, así como las acciones metodológicas para orientarlos; este conjunto de procesos se conoce como currículo. A propósito de lo anterior, Sacristán (2007) refiere el currículo como una construcción en la que convergen varios elementos, veamos:

El curriculum como proyecto concretado en un plan construido y ordenado hace relación a la conexión entre unos principios y una realización de los mismos, algo que ha de comprobarse y que en esa expresión práctica es donde concreta su valor. Es una práctica en la que se establece un diálogo, por decirlo así, entre agentes sociales, elementos técnicos, alumnos que reaccionan ante él, profesores que lo modelan, (p. 16)

En esta línea, el currículo puede ser entendido como el espacio en el que convergen los elementos, prácticas, apuestas y escenarios que se articulan en el establecimiento educativo y que tienen por objeto el proceso de aprendizaje de los estudiantes. Este proceso, está en el centro de las labores pedagógicas y didácticas que hacen los docentes, en tanto que éstos son los encargados de diseñar, orientar, gestionar e intencionar las prácticas pedagógicas de sus establecimientos, de acuerdo a los criterios, propósitos y fines a los que responde su currículo; de ahí su papel protagónico en su construcción y realimentación. En este mismo sentido los docentes agregan:

Como docentes tenemos la tarea de planear y evaluar esa planeación, es necesario revisar periódicamente lo que planteamos en el aula de clase y determinar con cuidado los ajustes; para tratar de incorporar las cosas que requieren los estudiantes y las exigencias derivadas del contexto. (D7)

Como señala el testimonio, la tarea es la acción permanente de reflexión sobre el contexto y sobre los saberes que toman relevancia para la participación de los estudiantes al interior del mismo; en palabras de los docentes participantes ello convoca la acción curricular. Esta acción curricular, pasa por distintas etapas en las que destacan la planeación, el desarrollo y la realimentación. El Siguiente testimonio ilustra lo anterior

Principios [que] tengo para preparar una clase. El primero tengo en cuenta la planeación del ministerio de educación porque con esos nos evalúan y evalúan al colegio, entonces por eso yo le doy la importancia. Lo segundo conocer el grupo y ver hasta donde le puede llevar y como lo que no tiene, hay un grado por ejemplo el grado sexto, no he sido capaz de algunos temas ubicarme con lo que el MEN propone para Él Lo otro es para que enseñarles ese tema de ciencias, ¿para qué? O sea, yo para que les enseño eso, siempre afortunadamente tengo la fortuna y el privilegio de tener todas las ciencias y tener como los colegios grandes y para que, a donde voy a llegar con eso y entonces para que enseño eso $Y$ lo otro es mirara que lenguaje le llego adherir y el interés del grupo a veces me hace cambiar el rumbo, el interés del grupo y también la dinámica del grupo tiene en ese momento. (D1)

La forma como se asumen esto tres momentos, hace la diferencia en la manera como el establecimiento educativo consolida las metas que ha construido, determina mecanismos para enfrentar las dificultades y construye nuevos horizontes hacia los cuales seguir avanzando.

Todas las acciones que agencia el establecimiento educativo ya sea desde el aula o las del orden institucional, comparten un momento en el que los actores alrededor del proceso determinan un plan de acción en el que se establecen propósitos, recursos, tiempos, entre otros aspectos.

A propósito de lo anterior, la UNESCO (2016) establece lo siguiente:

Un marco curricular normalmente debería incluir declaraciones sobre los valores subyacentes, las concepciones del aprendizaje, los principales objetivos, los propósitos y las tareas de la educación sobre la evolución de la cultura escolar, etc. Se trata de un documento básico de política en que se describe una serie de requisitos, reglamentos y recomendaciones que deben ser respetados por todas las partes 
interesadas en el sistema educativo, y que deberían orientar la labor de las escuelas, a los docentes y otros documentos curriculares, como los libros de texto y las guías docentes. (p. 27)

Como se señaló antes, hay tres fases en esta construcción en las que se centran las tensiones y alcances en cuanto al currículo se refiere, ellas son la planeación, el desarrollo y la realimentación. Para Sacristán (2007), estas tres fases constituyen un sistema cíclico, en permanente revisión por parte de la escuela, en el que reposan los propósitos, reflexiones, fracasos, metas y proyecciones del sistema escolar, por lo que se hace importante ahondar en las precisiones de cada una de estas etapas del currículo y la forma como se articulan en términos de la calidad: 1) La planificación escolar convoca pensar los propósitos, procedimientos, recursos y alcances de la acción didáctica que tiene lugar en el aula de clase y en la cual intervienen de manera directa docentes y estudiantes. Dicho proceso empieza en el momento que la comunidad educativa configura una hoja de ruta, en la que para Navarro (2008) significa deben articularse tres elementos: el contexto, la política pública y la gestión de aula. Esta afirmación de Navarro (2008) presenta coincidencias con lo expuesto en los hallazgos de la investigación:

Cuando el docente llega al aula de clase, no solo trae su experiencia y sus saberes. Uno viene con una lectura de lo que exige el medio, el conocimiento de lo que plantea la política pública y todo ello se articula con las voces de los estudiantes y en ese ejercicio dialógico la planeación se completa y puede ser desarrollada. (D6)

Es importante señalar que, en lo planteado por el docente, se muestra la planeación como un proceso inacabado, susceptible a la voz de otros actores escolares y al entorno educativo. De allí que el primer elemento a considerar en la fase de planeación está relacionado con el conocimiento del contexto escolar. Como ya se dijo, acercarse a una noción de contexto desde el ámbito curricular, implica trasgredir las barreras del espacio físico y encarar el terreno de las interacciones (políticas, económicas, culturales), las dinámicas discursivas y culturales de la comunidad, entre otros elementos.

El segundo componente de la fase de planeación está relacionado con los criterios de la política pública, los cuales además de complementar las construcciones derivadas de ese primer encuentro con el contexto, integran los elementos que la nación a través de las entidades gubernamentales ha privilegiado para la construcción de país. Sin embargo, la política pública exige para su materialización al interior del aula de clase del profesor. Toda vez, que la política solo determina unos marcos de referencia, un horizonte al cual llegar; pero es el maestro el que hace el encuadre didáctico determinando porqué, como, para qué y con quienes se construye ese saber. Esto se ve más claro en los comentarios de uno de los participantes, veamos:

Los lineamientos, estándares y criterios de calidad solo son orientaciones generales. La tarea del docente es armar el rompecabezas; no se trata de escoger entre una u otra o peor aún guiarse por lo que diga el libro de texto o el compañero con mayor experiencia. Se trata de un ejercicio dialéctico en que la norma se articula a la experiencia del docente, a la voz del estudiante y al contexto escolar. (D2)

El tercer componente de la etapa de planeación está asociado a las acciones directas que agencia el docente con sus estudiantes durante la clase. Es un espacio para configurar un marco metodológico para el hacer en el aula, una serie de orientaciones que den cuenta de los propósitos, tiempos, recursos y tareas de clase. En esta construcción, es fundamental favorecer el uso de configuraciones didácticas dinámicas, integradoras y flexibles que integren a los aprendizajes disciplinares experiencias en relación con los territorios, los problemas de las comunidades y las situaciones socialmente relevantes para los estudiantes; hacerlo contribuye a que la fase de planeación se proyecte sobre la acción de aula, las situaciones, personas y saberes implicados en ella. 
Una segunda fase que propone Sacristan (2008) es la implementación, la misma que se entiende como el conjunto de acciones didácticas que emergen durante el proceso de aprendizaje y cuyo propósito es suscitar un encuentro con los saberes que ha privilegiado la escuela y que se espera los estudiantes puedan construir en relación con los espacios que habitan, las maneras como leen su cultura y sus perspectivas de vida. Lo anterior obliga al establecimiento educativo al acompañamiento de lo que sucede en el aula, a sugerir variaciones metodológicas o conceptuales cuando ello sea necesario e incluso, a pensar otros caminos cuando la acción de aula se desdibuja frente a los propósitos de la misma. Es precisamente lo referido a las adecuaciones in situ uno de los elementos que resalta en los testimonios de los participantes, veamos:

Es indiscutible que el punto de partida para la clase es la planeación y esa especie de guión que construye el docente para el encuentro en el aula. Sin embargo, por tratarse la educación de un proceso social y evidentemente cultural es posible que ese guión deba ser modificado parcialmente e incluso en su totalidad durante el desarrollo de la clase y eso de ninguna manera es un fracaso, es más bien una oportunidad para acceder a los saberes desde otras perspectivas. (D4)

Es aquí cuando el acompañamiento del docente, juega un papel vital dentro de la implementación curricular del establecimiento educativo. Su tarea entonces es asistir de manera permanente las situaciones de aprendizaje en el aula de clase, brindar espacios para reflexionar en torno a lo que pasa, mediar en los conflictos que allí tienen lugar, proponer diversas maneras de enfrentarlos y convertirlos en oportunidades para el mejoramiento y gestionar las acciones necesarias para propender por los aprendizajes de los estudiantes.

Como tercera y última fase Sacristán (2008) propone la realimentación. Esta fase del currículo debe ser asumida por todos los integrantes de la comunidad educativa, es una tarea colectiva en la que cada uno de los sujetos escolares realiza un balance de la acción educativa en general, de los alcances obtenidos, las dificultades halladas y los retos que debe encarar el establecimiento. Se trata entonces de dar una mirada integral al currículo escolar, para lo que podría ser útil retomar además de los desempeños institucionales y los testimonios de la comunidad educativa, insumos del orden nacional como los resultados en las pruebas externas, los procesos de autoevaluación internos, entre otros elementos que contribuyan al análisis del currículo institucional y posibilitan algunas comprensiones alrededor del mismo.

Son esas comprensiones las que darán pie para explorar maneras alternativas para asumir la implementación del currículo en el establecimiento educativo, implementar mecanismos que permitan superar las dificultades evidenciadas, convocar a otras instituciones para enriquecer la discusión y fortalecer los procesos misionales del establecimiento educativo en términos de los aprendizajes que los estudiantes demandan y necesitan para participar de las dinámicas de sus comunidades, empoderarse de las mismas e incluso transformarlas, veamos:

Caminar hacia la calidad, implica la revisión permanente de lo que pasa en la clase. Es importante que los materiales, los textos y las actividades sean revisados tras cada una de las sesiones; los muchachos cambian, la tecnología se actualiza, es imposible que el maestro y lo que hace para enseñar no lo hagan. (D2)

Como lo señala el testimonio el trabajo docente es crucial, aquí se trata de configurar espacios para el diálogo y la reflexión sobre la práctica pedagógica en el establecimiento educativo, espacios que permitan alinear la gestión de aula con los propósitos del establecimiento, el contexto escolar, el currículo nacional y la voz de la comunidad, solo de esta unión puede desprenderse verdaderos procesos de cualificación y mejoramiento.

\section{Discusión}

En esa intención de adaptar el currículo a las necesidades e intereses de los estudiantes, como producto de la reflexión y la lectura del contexto, los maestros buscan las estrategias, que conllevan a tener logros en los 
procesos de calidad, enmarcadas bajo la perspectiva en la que expone la necesidad de la creación de un currículo basado en la práctica que se vive en la cotidianidad, con la intencionalidad de desarrollar estrategias que confluyen al interior del aula y que consideran un nuevo modo de evidenciar el aprendizaje que en ocasiones trasciende hacia la deconstrucción de las prácticas para el logro de aprendizajes significativos. Como se puede evidenciar en los testimonios presentados. Por otra parte, y sin lugar a dudas este ejercicio de apropiarse de la realidad y de adaptar las políticas educativas a las necesidades de las regiones, es un ejercicio necesario y urgente para el maestro sujeto político, quien en casos como estos se enfrenta a las propuestas de los modelos curriculares globalizantes y planes curriculares que pretenden homogenizar y centralizar la Educación (Peláez y Usma, 2017). De ahí que el maestro en este sentido siente que navega en contracorriente, frente a los postulados y lineamientos nacionales. Lo anterior nos lleva a entender e interpretar que la calidad en la educación para los maestros sujetos del estudio, es relacionar las experiencias de los maestros en el aula como producto de la lectura del contexto social y local. Contreras (2001) manifiesta que contrario a la reflexión está la racionalidad técnica, es decir, la manera tradicional de ver la enseñanza, en la cual hay una dicotomía entre teoría y práctica; y que ésta es limitada. Según él, tal separación deja de lado las incertidumbres, conflictos y dilemas que el maestro debe afrontar en su labor; deja de lado aquello que es imprevisto e inesperado en la conceptualización de la actuación profesional del docente. De este modo y ante las exigencias de la situación incompatible, ante los conflictos de valor, ante la incertidumbre de las consecuencias de determinadas actuaciones, no es sólo un problema de técnicas lo que debe el maestro resolver, sino un asunto de qué es lo que está en juego.

La reflexión es un proceso, que según Schön (1983), puede tomar lugar bien sea durante la acción como también antes y después de ella, lo que él llama reflexión en la acción y reflexión sobre la acción, respectivamente. Reflexión en la acción implica una observación introspectiva deliberada en el momento que ocurre un percance, como lo evidencia el testimonio anterior. La reflexión sobre la acción, por su parte, ocurre cuando el proceso reflexivo toma lugar fuera de la acción, es decir, fuera del tiempo en el aula de clase. Ocurre cuando se reflexiona, anterior o posteriormente, acerca de los resultados no deseados en el momento de la acción. Zeichner (1993), por su parte, dice que el ejercicio de reflexión implica la enseñanza como un proceso inacabado, que este ejercicio nunca finaliza debido a que el maestro permanentemente debe renovarse en términos académicos, pues solo así podrá brindar lo mejor de sí al estudiante.

Lo anterior, permite inferir que el maestro es el garante de calidad en términos educativos y así lo afirma Quitián (2015) que la calidad "no es un juicio sobre el objeto o representación en sí mismos, sino respecto de las expectativas, necesidades y deseos que estos incitan en una persona o comunidad" (p. 267). Es así, que en los procesos de calidad es importante reconocer el valor que tienen los sujetos que conforman las Instituciones Educativas, debido a que son quienes tienen la oportunidad de construir estrategias, los que conocen las diferentes necesidades y expectativas tanto de los estudiantes como del contexto en general.

En coherencia con lo enunciado, indagar por la calidad en contexto implica necesariamente el carácter reflexivo del maestro para pensar con las particularidades del aula, de las necesidades de la Institución, de los intereses de los estudiantes; un maestro reflexivo detecta cuando algo no marcha y emprende acciones para la solución de lo encontrado. Al respecto Dewey (1933) como se cita en Tagle (2011) define "la reflexión como una cuidadosa, persistente y activa consideración de cualquier creencia o forma de conocimiento a la luz de los cimientos que la sustentan". (p. 211). Esta concepción es fundamental debido a que difiere de la idea de la reflexión como un acto pasivo e indaga, al contrario, por la reflexión como el proceso adecuado de pensar a partir de las situaciones problemáticas.

No es algo que pueda acotarse de manera precisa como han tratado de hacer algunos, y enseñarse como un conjunto de técnicas para uso de los maestros; por el contrario, implica comprender y perfeccionar el propio ejercicio docente que ha de arrancar de la reflexión sobre la propia experiencia, la experiencia de los otros y su 
entorno, con la intencionalidad de cuestionar y transformar no sólo el aula de clase sino también las estructuras inequitativas de la sociedad.

\section{Conclusiones}

Referir la calidad escolar es acercarse a un proceso dinámico, complejo y en el que intervienen distintos actores. Por su naturaleza social y cultural no responde a fórmulas únicas y muta en cada comunidad, región e institución; por lo que la generalización de estrategias y métodos para lograrla pueden convertirse en diásporas que en poco o nada impacten la realidad de las aulas. Sin embargo, existen algunas líneas que, si logran integrarse a las particularidades de cada territorio, de los actores escolares y a los marcos de referencia de país, pueden constituir oportunidades para transitar el horizonte del mejoramiento continuo. En un primer momento se ha señalado la relevancia que tiene el contexto dentro de los procesos de cualificación escolar, su lectura permanente contribuye a que los aprendizajes avancen en términos de la pertinencia, la asertividad y la construcción de competencias. Comprender el contexto y sus dinámicas presupone un requisito para el mejoramiento escolar, para la cualificación de los aprendizajes y para avanzar de una institucionalidad escolar arraigada a los territorios y sus prácticas.

Lo encontrado en esta investigación a la relación entre contexto escolar y calidad educativa debe entenderse en dos líneas; la primera, referida a las dinámicas propias del espacio escolar y la manera como se articulan las personas que lo habitan en dichas dinámicas, costumbres y relaciones culturales, las mismas que resultan fundamentales para la tarea escolar de formar con criterios de pertinencia, asertividad y competencia. El otro elemento que convoca al contexto es la infraestructura, en donde es necesario poner el mismo énfasis en la adecuación de espacios, dotación de escenarios y en las políticas públicas que acompañan las dotaciones. Es necesario, frente al propósito de la calidad, atender al contexto como una construcción de los actores escolares que rebasa las barreras de los factores físicos, pero que no niega los múltiples aportes de estos, que necesita del concurso de todos para integrase a los lineamientos de formación escolar y que junto al profesor y el currículo constituyen bastiones determinantes a la hora de entender lo que pasa en el aula de clase.

El factor que mayor relación evidencia con el desarrollo de los procesos asociados a la calidad, está asociado con la práctica pedagógica y con la capacidad del docente de comprender su quehacer, reinventarse sobre la práctica y cualificar su ejercicio a través de la formación permanente y la investigación. Sin duda, el docente y sus prácticas al interior del aula, constituyen un binomio indisoluble a la hora de asumir la gestión académico-pedagógica como referente para el mejoramiento escolar. A lo anterior se le suma, los hallazgos concerniente a la relación entre calidad educativa y la formación permanente del profesorado, donde es importante señalar que los procesos de cualificación van más allá de los estudios universitarios (pregrado, postgrado, formación continua), se trata de incentivar al interior de los establecimientos pequeñas comunidades de aprendizaje, que puedan a partir de los intereses de sus integrantes privilegiar temáticas, discusiones y espacios para la reflexión, estos espacios resultan determinantes en el ensamblaje de la calidad escolar.

En términos del currículo es necesario precisar que, aunque existen lineamientos de política pública al interior de las distintas sociedades escolarizadas, estos son de orden de indicativo; por lo que son un insumo más en la construcción didáctica. Dicha construcción es el resultado de la experiencia del docente, de su formación, de la lectura que hace de las dinámicas sociales y culturales del territorio y de su apuesta política para impactar la vida de sus estudiantes; el tránsito hacia el mejoramiento permanente es la suma de voluntades, todas ellas coordinadas por la voz del docente. 
Los procesos de calidad en los establecimientos educativos, tal y como se ha señalado están permeados por distintos factores y protagonistas, por lo que asumirlos como una tarea permanente en la escuela requiere ponerlos en la discusión cotidiana. Es urgente asumir la discusión alrededor de la calidad y su gestión, como una acción que además de ser permanente, convoca a todos los integrantes de la comunidad educativa; ya que como se ha expuesto, va más allá de la acción del docente y del direccionamiento escolar, implica que toda la comunidad configure una visión compartida de lo que quiere aprender, de cómo desea hacerlo y en cuáles escenarios espera participar con esos saberes.

\section{Referencias Bibliográficas}

Aguerrondo, I. (1993). La calidad de la educación: ejes para su definición y evaluación. Colección: la Educación, 116 (3). Recuperado de http://www.educoas.org/portal/bdigital/contenido/laeduca/laeduca_116/articulo4/index.aspx

Bourdieu, P. (1997). Capital Cultural, Escuela y Espacio Social. Siglo XXI Editores

Canales C, M. (2006) Metodologías de investigación social. Santiago de Chile. Lom Ediciones

Cano, García, E. (1998). Evaluación de la calidad educativa. Madrid, Editorial Muralla S.A.

Casanova, M. A. (2012). El diseño curricular como factor de calidad educativa. Revista Iberoamericana sobre Calidad, Eficacia y Cambio en Educación, 10,(4), pp. 7-20. Recuperado de https://revistas.uam.es/reice/article/view/2984/3204

Contreras, J. (2001). La autonomía del profesorado. Madrid: Morata.

Egido, Galvez, I. (2005). Reflexiones en torno a la evaluación de la calidad educativa. Tendencia pedagógica, 10, pp. 17-28. Recuperado de file:///C:/Users/usuario/Downloads/DialnetReflexionesEnTornoALaEvaluacionDeLaCalidadEducativ-1407961\%20(1).pdf

Galeano, M. E. (2004). Diseño de proyec-tos en la investigación cualitativa. Medellín:Fondo Editorial EAFIT.

Gallego Henao, A., Pelaez Henao, O. A., Pino Montoya, J. W., González Ramírez, R. A y Arroyave Taborda, L. M. (2020). Contexto, didáctica y reflexión: desafíos para la Calidad Educativa. Revista espacios, 41 (1), pp. 1-9. Recuperado de https://www.revistaespacios.com/a20v41n18/a20v41n18p06.pdf

Króll, G. (2004). El método de los estudios de caso. En Tarrés Maria Luisa; Oservar, escuchar y comprender sobre la tradición cualitativa en la investigación social. Colegio de México, México.

López Parra, H. J. (2002). Investigación cualitativa y participativa. Un enfoque histórico-hermenéutico y crítico social en Psicología y Educación Ambiental. Medellín, Colombia: Universidad Pontificia Bolivariana.

Navarro, M.J. (2008) Procesos de planificación y gestión de los centros docentes: proyectos educativos para la diversidad. En Revista Iberoamericana de Educación, Organización de Estados Iberoamericanos para la Educación, la Ciencia y la Cultura (OEI)

Peláez, O., y Usma, J. (2017). The crucial role of educational stakeholders in the appropriation of foreign language education policies: A case study. PROFILE Issues in Teachers' Professional Development, 19(2), 121-134.

Quitian, D. (2015). Del alumbramiento a la puber-tad: los estudios sociales del deporte en América Latina, en clave colombiana. En C.M. Salazar y M.A. Lara Hidalgo (compiladores), Mundial de futbol 
Brasil 2014. Transversalidades y cono-cimientos múltiples sobre el mega-evento global (pp. 25-40). Colima: Universidad de Colima.

Restrepo, G. (2017). Aproximación Cultural al Concepto de Territorio. En: Biblioteca Virtual Luis Angél Arango. Índice de artículos sobre geografía. Banco de la República. Recuperado de: http://www.banrepcultural.org/blaavirtual/geografia/geografia/aprox.htm.

Sacristán, G. J. (2007). El curriculum. Una reflexión sobre la práctica. Madrid. Editorial Morata.

Schön, D. (1983). El profesional reflexivo. Cómo piensan los profesionales cuando actúan. Barcelona: Paidós.

Tagle, T. (2011). El enfoque reflexivo en la formación docente. Revista calidad en la educación, (34), pp. 203215

UNESCO (2014). Informe Mundial de Educación Para Todos 2013-1014. París.

UNESCO (2016). Qué hace un currículo de calidad. En Reflexiones en progreso № 2 sobre Cuestiones fundamentales y actuales del currículo y el aprendizaje. Santiago de Chile, UNESCO-Oficina para América Latina y el Caribe.

UNESCO-OREALC (2016), Suficiencia, equidad y efectividad de la infraestructura escolar en América Latina según el TERCE, Santiago, Chile.

Zambrano Leal, A. (2005): Tres tipos de saber del profesor y competencias: una relación compleja. Educere, 10, (33), pp. 225-232. Recuperado de https://www.redalyc.org/pdf/356/35603303.pdf

Zeichner, K. (1993). El maestro como profesional reflexivo. Cuadernos de Pedagogía,(220), pp. 44-45.

Esta obra está bajo una Licencia Creative Commons Attribución-NoCommercial 4.0 International

$(\mathrm{Cc}) \mathbf{B Y}$-NC 